OPEN ACCESS

Edited by:

Roberto Esposito,

A.O. Ospedali Riuniti Marche Nord,

Italy

Reviewed by:

Abraham Z. Snyder,

Washington University in St. Louis,

United States

Darya Chyzhyk,

Inria Saclay - île-de-France, France

*Correspondence:

Massimo Filippi

filippi.massimo@hsr.it

Specialty section: This article was submitted to Brain Imaging Methods, a section of the journal Frontiers in Neuroscience

Received: 05 April 2019 Accepted: 07 June 2019

Published: 20 June 2019

Citation:

Filippi M, Spinelli EG, Cividini C and Agosta F (2019) Resting State

Dynamic Functional Connectivity in Neurodegenerative Conditions: A Review of Magnetic Resonance Imaging Findings.

Front. Neurosci. 13:657. doi: 10.3389/fnins.2019.00657

\section{Resting State Dynamic Functional Connectivity in Neurodegenerative Conditions: A Review of Magnetic Resonance Imaging Findings}

\author{
Massimo Filippi1,2,3*, Edoardo G. Spinelli ${ }^{1}$, Camilla Cividini and Federica Agosta ${ }^{1,3}$ \\ ${ }^{1}$ Neuroimaging Research Unit, Institute of Experimental Neurology, Division of Neuroscience, IRCCS San Raffaele Scientific \\ Institute, Milan, Italy, ${ }^{2}$ Neurology Unit, IRCCS San Raffaele Scientific Institute, Milan, Italy, ${ }^{3}$ Vita-Salute San Raffaele \\ University, Milan, Italy
}

In the last few decades, brain functional connectivity $(\mathrm{FC})$ has been extensively assessed using resting-state functional magnetic resonance imaging (RS-fMRI), which is able to identify temporally correlated brain regions known as RS functional networks. Fundamental insights into the pathophysiology of several neurodegenerative conditions have been provided by studies in this field. However, most of these studies are based on the assumption of temporal stationarity of RS functional networks, despite recent evidence suggests that the spatial patterns of RS networks may change periodically over the time of an $\mathrm{fMRI}$ scan acquisition. For this reason, dynamic functional connectivity (dFC) analysis has been recently implemented and proposed in order to consider the temporal fluctuations of FC. These approaches hold promise to provide fundamental information for the identification of pathophysiological and diagnostic markers in the vast field of neurodegenerative diseases. This review summarizes the main currently available approaches for dFC analysis and reports their recent applications for the assessment of the most common neurodegenerative conditions, including Alzheimer's disease, Parkinson's disease, dementia with Lewy bodies, and frontotemporal dementia. Critical state-of-the-art findings, limitations, and future perspectives regarding the analysis of $\mathrm{dFC}$ in these diseases are provided from both a clinical and a technical point of view.

\footnotetext{
Keywords: dynamic functional connectivity, fMRI, neurodegeneration, dementia, Alzheimer's disease, Parkinson's disease, Lewy bodies, frontotemporal dementia
}

\section{INTRODUCTION: FROM STATIC TO DYNAMIC FUNCTIONAL CONNECTIVITY}

Neurodegenerative diseases are characterized by a progressive loss of neurons associated with deposition of aberrant proteins, leading to alterations of the structural and functional properties of the brain (Kovacs, 2017). In the last decades, the non-invasive restingstate (RS) functional magnetic resonance imaging (RS-fMRI) technique has been widely applied in these clinical populations, to investigate more in depth the spatial topology and strength of interactions between brain networks (Smitha et al., 2017; Hohenfeld et al., 2018). 
Functional magnetic resonance imaging uses the bloodoxygenation-level dependent (BOLD) signal, which is sensitive to spontaneous neural activity. In particular, low-frequency oscillations $(<0.1 \mathrm{~Hz})$ of the BOLD signal are analyzed to obtain functional information of brain networks. Functional connectivity (FC) quantifies the temporal correlation of functional activation in different brain regions and can be expressed in terms of pairwise Pearson's correlation coefficients, covariance, or mutual information between time series, revealing specific networks (Smitha et al., 2017). FC has been recognized as an important biomarker for better understanding the pathophysiological mechanisms of numerous neurodegenerative diseases, including Alzheimer's disease (AD) (Filippi et al., 2017), Parkinson's disease (PD) (Baggio et al., 2015; Filippi et al., 2019), and frontotemporal dementia (FTD) (Filippi et al., 2017).

So far, the implicit hypothesis on which FC analysis has been based is the assumption of temporal stationarity of the functional interaction between connections. Considering the dynamic nature of brain activity, a novel approach is provided by dynamic functional connectivity $(\mathrm{dFC})$, which considers the temporal fluctuations of functional connections in faster timescales (Hutchison et al., 2013). Unlike conventional static FC, which is obtained from the correlation within an entire time series, dFC refers to the brain activity within sub-portions of time series (Menon and Krishnamurthy, 2019). Major efforts have been made to identify and analyze time-varying, but recurring, FC sub-patterns of coupling among brain regions, constituting the brain "chronnectome" (Calhoun et al., 2014).

The aim of this review is to describe the contribution of $\mathrm{dFC}$ studies in RS conditions for a better understanding of neurodegenerative diseases. We are going to focus on the most common approaches to analyze $\mathrm{dFC}$ and review recent findings in this field concerning $\mathrm{AD}, \mathrm{PD}$ and other parkinsonisms, and FTD. We conclude this work summarizing caveats, limitations and future perspectives regarding $\mathrm{dFC}$ analysis.

\section{METHODOLOGICAL OVERVIEW}

Several computational strategies have been implemented to characterize temporal and spatial variations of BOLD signal (Wee et al., 2016; Jie et al., 2018; Liu et al., 2018). The most common approach is provided by the sliding-window technique (Chen et al., 2016, 2017; de Vos et al., 2018; Diez-Cirarda et al., 2018), characterized by the selection of a time window - shorter than the whole-scan time - whose data points are used to calculate FC metrics. The window is shifted in time by a fixed number of data points, referred as step, which defines the overlap between two successive windows. The step duration ranges from one single data point to the length of the window (i.e., non-overlapping windows) (Jie et al., 2018; Park et al., 2018).

In combination with the sliding-window approach, several studies have applied clustering methods to identify reproducible, transient patterns and to evaluate the commonly used graph metrics, the dwell time, defined as the number of consecutive windows in a specific state, and the number of transitions between states (Allen et al., 2014).
Since a subject could be in more than one state at a given point, the concept of "meta-states" and meta-state measures has been introduced to intuitively characterize the dynamic fluidity in FC (Miller et al., 2016; Premi et al., 2019). Meta-state measures include the number of occupied meta-states, number of switches between meta-states, greatest distance between two meta-states and overall distance (Premi et al., 2019). Furthermore, the sliding-window approach can be integrated with the application of independent component analysis (ICA) to identify spatial maps in the windowed BOLD signal and assess variability or graph theoretical metrics (Jones et al., 2012; de Vos et al., 2018; Premi et al., 2019). The sliding-window approach can also be used jointly with classification algorithms to exploit the information resulting from patterns of dFC (Chen et al., 2016; Guo et al., 2017; Figure 1).

Alternative approaches to evaluate $\mathrm{dFC}$ are represented by time-frequency analysis, dynamic connectivity regression (DCR) and dynamic connectivity detection (DCD), which are datadriven techniques for detecting FC change points within RS FMRI time series, and derive dynamic information from such points (Xu and Lindquist, 2015).

\section{ALZHEIMER'S DISEASE}

Alzheimer's disease is the most common neurodegenerative cause of dementia (Alzheimer's, 2016) and has been extensively studied by means of advanced MRI techniques. The inclusion of RS fMRI into imaging protocols in AD has been particularly advantageous, as the difficulty to obtain subjects' cooperation could influence task-related fMRI results. Conspicuous evidence has shown decreased FC of the default mode network (DMN) across the $\mathrm{AD}$ continuum, including patients with full-blown $\mathrm{AD}$ dementia and amnestic mild cognitive impairment (MCI) (Greicius et al., 2004; Bai et al., 2008; Agosta et al., 2012; Koch et al., 2012). Decreased connectivity within the DMN - consisting of the posterior cingulate, inferior parietal, inferolateral temporal, anterior cingulate, prefrontal, and hippocampal regions - is often accompanied by increased connectivity in the attentional frontoparietal and salience networks, likely mirroring compensatory mechanisms (Agosta et al., 2012; Badhwar et al., 2017). Disconnection between posterior (i.e., posterior cingulate and parietal regions) and anterior DMN nodes (i.e., anterior cingulate and prefrontal regions) was found to cause relative decreased connectivity within the posterior DMN and increased connectivity within the anterior DMN (Jones et al., 2011). Functional rearrangements have demonstrated clinical usefulness for predicting conversion to AD in MCI patients (Bai et al., 2011; Petrella et al., 2011; Li et al., 2016).

Given the high consistency of these findings across static FC studies, AD represents a good candidate to apply dFC approaches to the field of neurodegenerative disorders, since capturing the evolving architecture of brain networks over short periods of time might provide further pathophysiological insights into these conditions, and eventually better diagnostic or prognostic indicators. The first study investigating $\mathrm{dFC}$ of $\mathrm{AD}$ patients examined changes over time of a modularity 


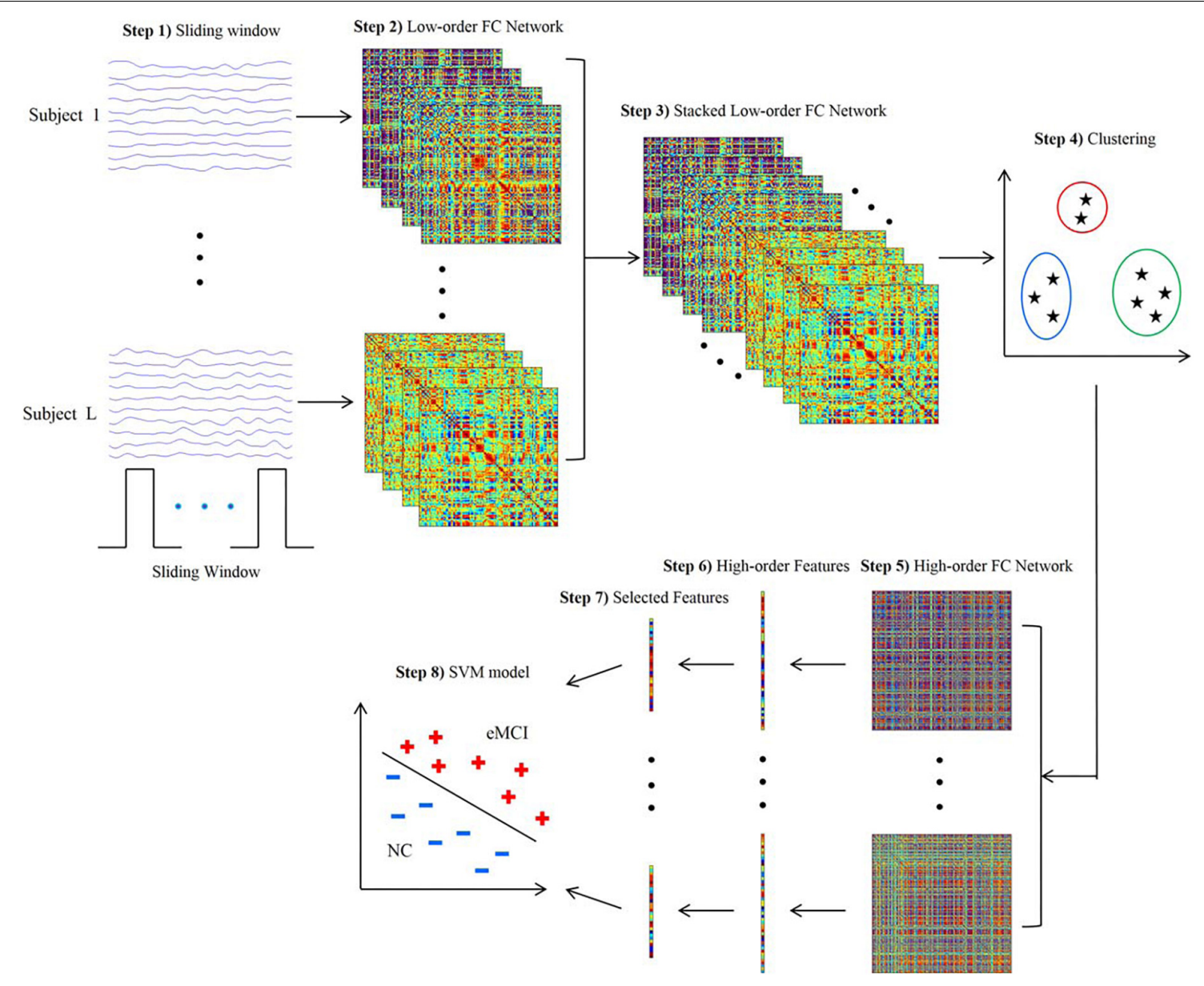

FIGURE 1 | Framework for construction of high-order functional connectivity (FC) network. (1) Partition of the RS-fMRI time series into multiple overlapping segments of subseries applying sliding-window technique; (2) collection of low-order FC matrices, one for each subseries; (3) stack of all matrices of all subjects together to obtain correlation time series for each element; (4) application of the clustering algorithm to group all the correlation time series; (5) construction of high-order FC network, considering the mean correlation time series for each cluster as vertex and the pairwise Pearson's correlation coefficient between each pair of vertices as weight; (6) calculation of local clustering coefficients; (7) selection of a discriminative feature subset from the local clustering coefficients; (8) implementation of support vector machine (SVM) model for classification. RS-fMRI, resting-state functional magnetic resonance imaging; FC, functional connectivity (reproduced with permission from Chen et al., 2016).

metric using a sliding-window analysis (Jones et al., 2012). The non-stationary nature of the brain modular organization was demonstrated and related with significant variations of the dwell time within different sub-network configurations of the DMN in subjects with $\mathrm{AD}$ dementia compared with healthy controls; specifically, $\mathrm{AD}$ patients spent less time in brain functional states with strong posterior DMN region contribution and more time in states with greater anterior DMN region contribution (Jones et al., 2012). A subsequent study investigated the evolution of $\mathrm{dFC}$ disruptions across the $\mathrm{AD}$ spectrum, showing alterations in patients with dementia compared to MCI and subjective cognitive decline (SCD) in terms of local dFC within the temporal, frontal-superior and default-mode networks; decreased global metastability between functional states was also found, supporting the hypothesis that oscillatory patterns are progressively altered over the AD continuum, eventually leading to a shrinkage of the "dynamic repertoire" (i.e., a smaller set of functional configurations) in the brain of AD patients (CordovaPalomera et al., 2017). Consistently, another study showed a progressive loss of whole-brain metastability according to the severity of cognitive impairment along the $\mathrm{AD}$ continuum, reaching statistical significance only in patients with dementia, when compared with healthy controls (Demirtas et al., 2017).

Researchers have also aimed to identify dFC alterations that may represent candidate non-invasive diagnostic biomarkers in the early stages of AD. A sparse temporal network-based framework has been tested for the classification of patients with early MCI by means of support vector machine (SVM) algorithms, yielding an accuracy of approximately $80 \%$ in the discrimination from healthy controls, compared with accuracies ranging 62-72\% using FC static approaches (Wee et al., 2016). Another recent study aimed to integrate both temporal and spatial properties of dFC networks for the classification of early and late MCI patients (Jie et al., 2018). Accuracies of 


\section{A Group-specific cluster centroids State I}

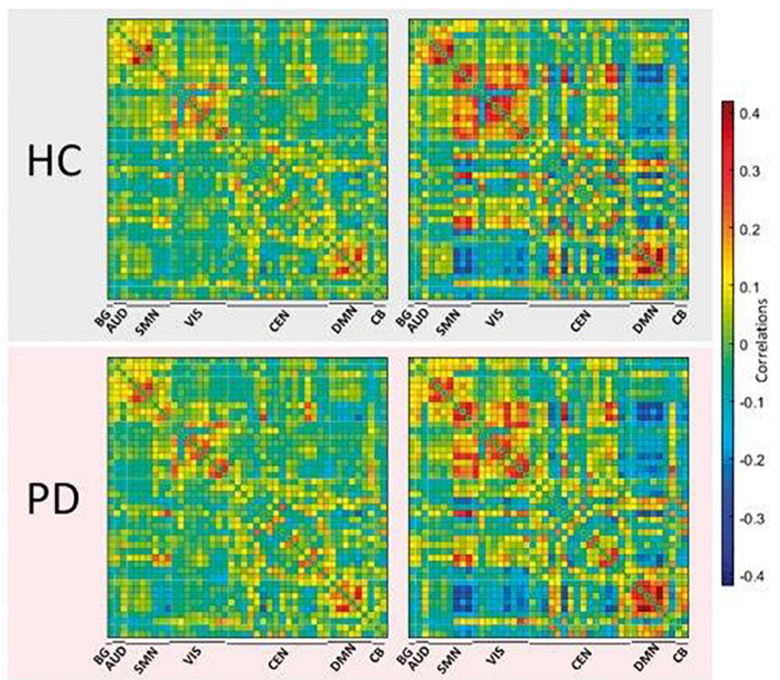

B Functional connectivity

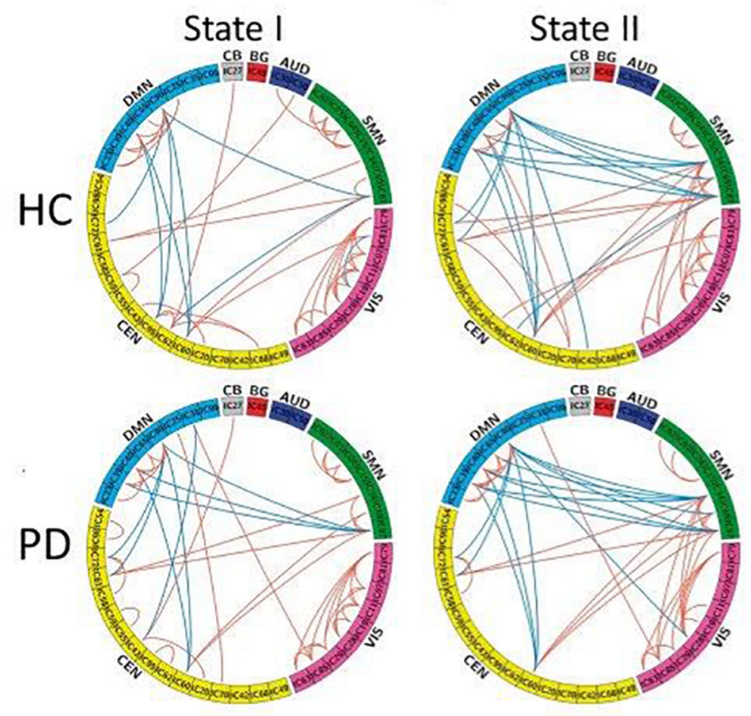

FIGURE 2 | Functional connectivity state results. (A) Group-specific cluster centroid for each state, averaged across subject-specific median cluster centroids of each group [percentage of total occurrences for stage I and II: $83.4 \%$ and $16.6 \%$ in the healthy controls (HC) and $70.8 \%$ and $29.2 \%$ in the Parkinson's disease (PD) group, respectively]. (B) Functional connectivity in each state is shown for healthy controls and Parkinson's disease groups, representing the $5 \%$ of the functional connectivity network with the strongest connections. BG, basal ganglia; AUD, auditory; SMN, sensorimotor; VIS, visual; CEN, cognitive executive; CB, cerebellar network (reproduced with permission from Kim et al., 2017).

approximately 78\% were obtained when SVM algorithms were trained based on the dFC patterns of components of the DMN and temporal and cerebellar regions (Jie et al., 2018). An attempt to combine multiple dFC parameters for an automated classification of early MCI patients was recently proposed by applying a tensor model of spatio-temporal BOLD signal to each voxel of the white matter (WM), to be integrated with the information provided by the FC of grey matter (GM) regions (Chen et al., 2017). Such combined GM and WM approach yielded an accuracy of almost $79 \%$ in discriminating early MCI patients from healthy subjects, compared with $74 \%$ provided by GM dFC measures alone (Chen et al., 2017). Another promising approach is the clustering of standard-correlation time series for all pairs of brain regions (i.e., the classic "low-order" FC networks) into a smaller set of "high-order" dFC networks according to their intrinsic common patterns. The combination of high-order dFC with the conventional low-order analysis allowed SVM-based discrimination of early MCI patients from healthy subjects with an accuracy of $88 \%$, outperforming other previous approaches (Chen et al., 2016; Figure 1).

\section{PARKINSON'S DISEASE}

Parkinson's disease (PD) is the second most common neurodegenerative disorder and is characterized by dopamine depletion in the nigro-striatal system leading to progressive functional impairment (Poewe et al., 2017). Widespread functional rearrangements related to the development of motor and non-motor symptoms occur over the clinical progression in PD patients (Filippi et al., 2019). Several RS fMRI studies have identified alterations of the cerebello-thalamo-cortical circuit as a key hallmark of PD (Helmich et al., 2010; Hacker et al., 2012; Agosta et al., 2014; Akram et al., 2017), with reduced activation of the posterior putamen correlating with motor impairment as the most consistent finding (Herz et al., 2014). Furthermore, disrupted FC in the DMN, fronto-parietal, salience and associative visual networks has been linked to the development of cognitive deficits in PD (Amboni et al., 2015; Baggio et al., 2015; Putcha et al., 2015; Zhan et al., 2018). Particularly, normal decoupling between the DMN and fronto-parietal networks was reduced in PD patients with MCI (PD-MCI) (Baggio et al., 2015; Putcha et al., 2015), and FC alterations within the DMN were able to predict subsequent cognitive decline in cognitively unimpaired PD patients (van Eimeren et al., 2009; Tessitore et al., 2012; Putcha et al., 2015).

The first study assessing the dynamic functional properties of RS networks in PD patients identified two main FC configurations: a more frequent and strongly segregated state (defined as "State I") and a less frequent, more integrated "State II" (Kim et al., 2017). Compared with healthy subjects, PD patients showed a significant decrease of dwell time in State I, with a proportional increase of dwell time in State II that was correlated with the severity of motor symptoms, indicating that the loss of functional segregation between brain networks might represent a key element in PD pathogenesis (Kim et al., 2017; Figure 2). To eliminate the possible influence of long-term dopaminergic therapy, dFC alterations were also 
assessed in early stage, drug-naïve PD patients, who showed decreased switching rate between dynamic states correlating with disease severity, supporting the view that a limited dynamic range of whole-brain FC might represent an early PD marker (Cordes et al., 2018; Zhuang et al., 2018). Another recent study focused on the spatial configuration of dFC alterations within two homogeneous subunits of the putamen of PD subjects, demonstrating a degradation of subregional specificity between the anterior and posterior putaminal subunits that was related with disease severity (Liu et al., 2018).

Along with motor impairment, $\mathrm{dFC}$ has also provided significant information regarding the underpinnings of cognitive symptoms in PD. Decreased dwell time in a more segregated state and increased state transitions have been demonstrated in PD-MCI patients compared with healthy controls, configuring a pattern that PD patients with normal cognition lacked (DiezCirarda et al., 2018). Increased dFC within the dorsal-attention network was found to predict attention performance in $\mathrm{PD}$ patients (Madhyastha et al., 2015), whereas a positive correlation between $\mathrm{dFC}$ of the DMN and performance on a visuospatial memory task has also been recently reported (Engels et al., 2018).

\section{OTHER NEURODEGENERATIVE CONDITIONS}

To date, most dFC studies assessing patients with neurodegenerative conditions have focused on the two most common diseases, i.e., $\mathrm{AD}$ and $\mathrm{PD}$. Considering also the novelty of these approaches, evidence regarding other pathological entities is currently scarce, but in rapid development.

Dementia with Lewy bodies (DLB) is among the most common causes of dementia after $\mathrm{AD}$, and is characterized by cognitive fluctuations, parkinsonism, and visual hallucinations (McKeith et al., 2017). Classic RS-fMRI static studies have shown FC reductions in widespread brain networks in DLB subjects, with desynchronization of cortical and subcortical areas within the attention-executive networks correlating with cognitive fluctuations (Lowther et al., 2014; Peraza et al., 2014). Considering the transient nature of some of the main features of DLB (i.e., cognitive fluctuations and hallucinations), dFC studies are expected to provide fundamental insights into the pathophysiology of this disease. Indeed, $\mathrm{dFC}$ has demonstrated significant differences in DLB patients compared with healthy subjects in visual (i.e., the occipito-parieto-frontal and medial occipital networks) and attentional networks (i.e., the right fronto-parietal control network), which also showed decreased mutual dependency, suggesting that temporal disconnection between these networks might be relevant for DLB pathogenesis (Sourty et al., 2016).

Frontotemporal dementia is another frequent neurodegenerative condition encompassing a wide range of clinical presentations, including behavioral, executive, language and motor deficits (Olney et al., 2017). To our knowledge, no study has assessed dFC alterations in patients with FTD. However, a recent work focused on presymptomatic carriers of FTD-causing mutations (Premi et al., 2019), being FTD an inherited autosomal disorder in $30-40 \%$ of cases (Rohrer and Warren, 2011). Mutation carriers showed lower number of meta-states, decreased switching rate between meta-states and shorter meta-state total distance compared with healthy controls, demonstrating that a reduced dynamic fluidity and restricted dynamic range in brain functional "chronnectome" is an early event in the development of FTD (Premi et al., 2019). The assessment of such alterations in the phase which is closest to clinical conversion might be a promising research field for the development of biomarkers to be used in clinical trials of FTD.

\section{CAVEATS, LIMITATIONS AND FUTURE DIRECTIONS}

Based on the evidence here reviewed, dFC studies have shed new light on the pathophysiological alterations underlying the most common neurodegenerative diseases. However, important concerns remain regarding the possible influence of vigilance fluctuations during the fMRI scan. Although patients are routinely instructed to stay awake for the whole scan duration, sleep disturbances are frequent clinical features of dementias and parkinsonian syndromes (Malhotra, 2018), and fluctuating alertness will affect FC (Tagliazucchi et al., 2012; Haimovici et al., 2017). This important issue would be overcome by simultaneous EEG-fMRI acquisition (Tagliazucchi and Laufs, 2014; Allen et al., 2018), although this approach is technically challenging and has not been explored in neurodegenerative conditions yet.

Some technical caveats also need to be considered. The slidingwindow technique has been repeatedly applied because of its analytical simplicity and easy implementation: in most studies, the number of BOLD signal subseries $\mathrm{K}$ is decided based on the window length $\mathrm{W}$, the number of temporal image volumes $\mathrm{N}$ and the step, according to the formulation $\mathrm{K}=[(\mathrm{N}-\mathrm{W}) / \mathrm{s}]$ +1 (Chen et al., 2016; Wee et al., 2016; Guo et al., 2017). However, the window length, as well as the step parameter, are matter of debate: choosing a short window could increase the risk of misleading spurious fluctuations, while choosing a long window could fail to identify state transitions (Preti et al., 2017). A trade-off must be reached: at present, different studies suggested a window length of 30-60 s as sufficient for detecting dFC changes (Jones et al., 2012; Diez-Cirarda et al., 2018; Premi et al., 2019), even though some studies opted for longer windows (Chen et al., 2016; Wee et al., 2016). An alternative way to find the optimum window length is represented by the timefrequency analysis (Cordes et al., 2018). The step parameter is also chosen arbitrarily, commonly ranging one to two volumes for overlapping windows (Quevenco et al., 2017; Liu et al., 2018), although a few studies adopted non-overlapping windows (Jie et al., 2018; Park et al., 2018). Another crucial point is the choice of window shape: rectangular, modulated rectangular or tapered windows are the most used (Diez-Cirarda et al., 2018; Premi et al., 2019).

Beyond these methodological aspects, there are still some unsolved questions regarding dFC. Neurobiological underpinnings and mechanisms of dynamic states have to be 
clarified (Smith et al., 2011), as the possibility that reported changes may be driven by signal noise or sampling variability needs to be considered (Laumann et al., 2017). Indeed, the fluctuations in the sliding-window correlation time series can be associated to $\mathrm{dFC}$ or simply generated by random noise, so more complex statistical models are required to deal with this issue (Hindriks et al., 2016). The artifact problem that applies to conventional resting-state fMRI is also a crucial aspect for dFC analysis (Nalci et al., 2019), as the BOLD signal is sensitive to non-stationary physiological processes, such as head motion (Power et al., 2014) and blood partial pressure of carbon dioxide (pCO2) due to respiration (Power et al., 2018). Moreover, important factors to take into account for a correct interpretation of $\mathrm{dFC}$ results are the selection of the a priori atlas or ICA algorithm used to obtain regions of interest and the assessment of specific FC metrics. Finally, the reliability and reproducibility of dFC patterns are still a challenge, although some efforts have been made on solving this issue (Abrol et al., 2017).

\section{REFERENCES}

Abrol, A., Damaraju, E., Miller, R. L., Stephen, J. M., Claus, E. D., Mayer, A. R., et al. (2017). Replicability of time-varying connectivity patterns in large resting state fMRI samples. Neuroimage 163, 160-176. doi: 10.1016/j.neuroimage.2017. 09.020

Agosta, F., Caso, F., Stankovic, I., Inuggi, A., Petrovic, I., Svetel, M., et al. (2014). Cortico-striatal-thalamic network functional connectivity in hemiparkinsonism. Neurobiol. Aging 35, 2592-2602. doi: 10.1016/j. neurobiolaging.2014.05.032

Agosta, F., Pievani, M., Geroldi, C., Copetti, M., Frisoni, G. B., and Filippi, M. (2012). Resting state fMRI in Alzheimer's disease: beyond the default mode network. Neurobiol. Aging 33, 1564-1578. doi: 10.1016/j.neurobiolaging.2011. 06.007

Akram, H., Wu, C., Hyam, J., Foltynie, T., Limousin, P., De Vita, E., et al. (2017). 1-Dopa responsiveness is associated with distinctive connectivity patterns in advanced Parkinson's disease. Mov. Disord. 32, 874-883. doi: 10.1002/mds. 27017

Allen, E. A., Damaraju, E., Eichele, T., Wu, L., and Calhoun, V. D. (2018). EEG signatures of dynamic functional network connectivity states. Brain Topogr. 31, 101-116. doi: 10.1007/s10548-017-0546-542

Allen, E. A., Damaraju, E., Plis, S. M., Erhardt, E. B., Eichele, T., and Calhoun, V. D. (2014). Tracking whole-brain connectivity dynamics in the resting state. Cereb. Cortex 24, 663-676. doi: 10.1093/cercor/bhs352

Alzheimer's, A. (2016). 2016 Alzheimer's disease facts and figures. Alzheimers Dement 12, 459-509. doi: 10.1016/j.jalz.2016.03.001

Amboni, M., Tessitore, A., Esposito, F., Santangelo, G., Picillo, M., Vitale, C., et al. (2015). Resting-state functional connectivity associated with mild cognitive impairment in Parkinson's disease. J. Neurol. 262, 425-434. doi: 10.1007/ s00415-014-7591-7595

Badhwar, A., Tam, A., Dansereau, C., Orban, P., Hoffstaedter, F., and Bellec, P. (2017). Resting-state network dysfunction in Alzheimer's disease: a systematic review and meta-analysis. Alzheimers Dement 8, 73-85. doi: 10.1016/j.dadm. 2017.03.007

Baggio, H. C., Segura, B., Sala-Llonch, R., Marti, M. J., Valldeoriola, F., Compta, Y., et al. (2015). Cognitive impairment and resting-state network connectivity in Parkinson's disease. Hum. Brain Mapp. 36, 199-212. doi: 10.1002/hbm.22622

Bai, F., Liao, W., Watson, D. R., Shi, Y., Wang, Y., Yue, C., et al. (2011). Abnormal whole-brain functional connection in amnestic mild cognitive impairment patients. Behav. Brain Res. 216, 666-672. doi: 10.1016/j.bbr.2010.09.010

Bai, F., Zhang, Z., Yu, H., Shi, Y., Yuan, Y., Zhu, W., et al. (2008). Default-mode network activity distinguishes amnestic type mild cognitive impairment from

\section{CONCLUSION}

Assessing $\mathrm{dFC}$ is a promising way to better understand neurodegenerative processes and investigate novel disease diagnostic and prognostic biomarkers. However, future developments are needed to rule out the influence of vigilance fluctuations, overcome the limitations of the sliding-window approach - possibly using other methods as time-frequency analysis -, identify the most informative dFC metrics, and minimize artifacts by means of adequate preprocessing, so as to be more confident in the description and interpretation of these findings.

\section{AUTHOR CONTRIBUTIONS}

MF contributed to the study concept and acted as study supervisor. All authors contributed to writing, reading, and approving the final version of the manuscript.

healthy aging: a combined structural and resting-state functional MRI study. Neurosci. Lett. 438, 111-115. doi: 10.1016/j.neulet.2008.04.021

Calhoun, V. D., Miller, R., Pearlson, G., and Adali, T. (2014). The chronnectome: time-varying connectivity networks as the next frontier in fMRI data discovery. Neuron 84, 262-274. doi: 10.1016/j.neuron.2014.10.015

Chen, X., Zhang, H., Gao, Y., Wee, C. Y., Li, G., Shen, D., et al. (2016). High-order resting-state functional connectivity network for MCI classification. Hum. Brain Mapp. 37, 3282-3296. doi: 10.1002/hbm.23240

Chen, X., Zhang, H., Zhang, L., Shen, C., Lee, S. W., and Shen, D. (2017). Extraction of dynamic functional connectivity from brain grey matter and white matter for MCI classification. Hum. Brain Mapp. 38, 5019-5034. doi: 10.1002/hbm.23711

Cordes, D., Zhuang, X., Kaleem, M., Sreenivasan, K., Yang, Z., Mishra, V., et al. (2018). Advances in functional magnetic resonance imaging data analysis methods using empirical mode decomposition to investigate temporal changes in early Parkinson's disease. Alzheimers Dement 4, 372-386. doi: 10.1016/j.trci. 2018.04.009

Cordova-Palomera, A., Kaufmann, T., Persson, K., Alnaes, D., Doan, N. T., Moberget, T., et al. (2017). Disrupted global metastability and static and dynamic brain connectivity across individuals in the Alzheimer's disease continuum. Sci. Rep. 7:40268. doi: 10.1038/srep40268

de Vos, F., Koini, M., Schouten, T. M., Seiler, S., van der Grond, J., Lechner, A., et al. (2018). A comprehensive analysis of resting state fMRI measures to classify individual patients with Alzheimer's disease. Neuroimage 167, 62-72. doi: 10.1016/j.neuroimage.2017.11.025

Demirtas, M., Falcon, C., Tucholka, A., Gispert, J. D., Molinuevo, J. L., and Deco, G. (2017). A whole-brain computational modeling approach to explain the alterations in resting-state functional connectivity during progression of Alzheimer's disease. Neuroimage Clin. 16, 343-354. doi: 10.1016/j.nicl.2017. 08.006

Diez-Cirarda, M., Strafella, A. P., Kim, J., Pena, J., Ojeda, N., Cabrera-Zubizarreta, A., et al. (2018). Dynamic functional connectivity in Parkinson's disease patients with mild cognitive impairment and normal cognition. Neuroimage Clin. 17, 847-855. doi: 10.1016/j.nicl.2017.12.013

Engels, G., Vlaar, A., McCoy, B., Scherder, E., and Douw, L. (2018). Dynamic functional connectivity and symptoms of Parkinson's disease: a resting-state fMRI study. Front. Aging Neurosci. 10:388. doi: 10.3389/fnagi.2018.00388

Filippi, M., Basaia, S., Canu, E., Imperiale, F., Meani, A., Caso, F., et al. (2017). Brain network connectivity differs in early-onset neurodegenerative dementia. Neurology 89, 1764-1772. doi: 10.1212/WNL.0000000000004577

Filippi, M., Sarasso, E., and Agosta, F. (2019). Resting-state functional MRI in Parkinsonian syndromes. Mov. Disord. Clin. Pract. 6, 104-117. doi: 10.1002/ mdc3.12730 
Greicius, M. D., Srivastava, G., Reiss, A. L., and Menon, V. (2004). Defaultmode network activity distinguishes Alzheimer's disease from healthy aging: evidence from functional MRI. Proc. Natl. Acad Sci. U.S.A. 101, 4637-4642. doi: 10.1073/pnas.0308627101

Guo, H., Liu, L., Chen, J., Xu, Y., and Jie, X. (2017). Alzheimer classification using a minimum spanning tree of high-order functional network on fMRI dataset. Front. Neurosci. 11:639. doi: 10.3389/fnins.2017.00639

Hacker, C. D., Perlmutter, J. S., Criswell, S. R., Ances, B. M., and Snyder, A. Z. (2012). Resting state functional connectivity of the striatum in Parkinson's disease. Brain 135(Pt 12), 3699-3711. doi: 10.1093/brain/ aws 281

Haimovici, A., Tagliazucchi, E., Balenzuela, P., and Laufs, H. (2017). On wakefulness fluctuations as a source of BOLD functional connectivity dynamics. Sci. Rep. 7:5908. doi: 10.1038/s41598-017-06389-6384

Helmich, R. C., Derikx, L. C., Bakker, M., Scheeringa, R., Bloem, B. R., and Toni, I. (2010). Spatial remapping of cortico-striatal connectivity in Parkinson's disease. Cereb. Cortex 20, 1175-1186. doi: 10.1093/cercor/ bhp 178

Herz, D. M., Eickhoff, S. B., Lokkegaard, A., and Siebner, H. R. (2014). Functional neuroimaging of motor control in Parkinson's disease: a meta-analysis. Hum. Brain Mapp. 35, 3227-3237. doi: 10.1002/hbm.22397

Hindriks, R., Adhikari, M. H., Murayama, Y., Ganzetti, M., Mantini, D., Logothetis, N. K., et al. (2016). Can sliding-window correlations reveal dynamic functional connectivity in resting-state fMRI? Neuroimage 127, 242-256. doi: 10.1016/j. neuroimage.2015.11.055

Hohenfeld, C., Werner, C. J., and Reetz, K. (2018). Resting-state connectivity in neurodegenerative disorders: is there potential for an imaging biomarker? Neuroimage Clin. 18, 849-870. doi: 10.1016/j.nicl.2018.03.013

Hutchison, R. M., Womelsdorf, T., Allen, E. A., Bandettini, P. A., Calhoun, V. D., Corbetta, M., et al. (2013). Dynamic functional connectivity: promise, issues, and interpretations. Neuroimage 80, 360-378. doi: 10.1016/j.neuroimage.2013. 05.079

Jie, B., Liu, M., and Shen, D. (2018). Integration of temporal and spatial properties of dynamic connectivity networks for automatic diagnosis of brain disease. Med. Image Anal. 47, 81-94. doi: 10.1016/j.media.2018. 03.013

Jones, D. T., Machulda, M. M., Vemuri, P., McDade, E. M., Zeng, G., Senjem, M. L., et al. (2011). Age-related changes in the default mode network are more advanced in Alzheimer disease. Neurology 77, 1524-1531. doi: 10.1212/WNL. 0b013e318233b33d

Jones, D. T., Vemuri, P., Murphy, M. C., Gunter, J. L., Senjem, M. L., Machulda, M. M., et al. (2012). Non-stationarity in the "resting brain's" modular architecture. PLoS One 7:e39731. doi: 10.1371/journal.pone.003 9731

Kim, J., Criaud, M., Cho, S. S., Diez-Cirarda, M., Mihaescu, A., Coakeley, S., et al. (2017). Abnormal intrinsic brain functional network dynamics in Parkinson's disease. Brain 140, 2955-2967. doi: 10.1093/brain/awx233

Koch, W., Teipel, S., Mueller, S., Benninghoff, J., Wagner, M., Bokde, A. L., et al. (2012). Diagnostic power of default mode network resting state fMRI in the detection of Alzheimer's disease. Neurobiol. Aging 33, 466-478. doi: 10.1016/j. neurobiolaging.2010.04.013

Kovacs, G. G. (2017). Concepts and classification of neurodegenerative diseases. Handb. Clin. Neurol. 145, 301-307. doi: 10.1016/B978-0-12-802395-2. 00021-23

Laumann, T. O., Snyder, A. Z., Mitra, A., Gordon, E. M., Gratton, C., Adeyemo, B., et al. (2017). On the stability of BOLD fMRI correlations. Cereb. Cortex 27, 4719-4732. doi: 10.1093/cercor/bhw265

Li, Y., Wang, X., Li, Y., Sun, Y., Sheng, C., Li, H., et al. (2016). Abnormal restingstate functional connectivity strength in mild cognitive impairment and its conversion to Alzheimer's Disease. Neural. Plast. 2016:4680972. doi: 10.1155/ 2016/4680972

Liu, A., Lin, S. J., Mi, T., Chen, X., Chan, P., Wang, Z. J., et al. (2018). Decreased subregional specificity of the putamen in Parkinson's disease revealed by dynamic connectivity-derived parcellation. Neuroimage Clin. 20, 1163-1175. doi: $10.1016 /$ j.nicl.2018.10.022

Lowther, E. R., O’Brien, J. T., Firbank, M. J., and Blamire, A. M. (2014). Lewy body compared with Alzheimer dementia is associated with decreased functional connectivity in resting state networks. Psychiatry Res. 223, 192-201. doi: 10. 1016/j.pscychresns.2014.06.004

Madhyastha, T. M., Askren, M. K., Boord, P., and Grabowski, T. J. (2015). Dynamic connectivity at rest predicts attention task performance. Brain Connect. 5, 45-59. doi: 10.1089/brain.2014.0248

Malhotra, R. K. (2018). Neurodegenerative disorders and sleep. Sleep Med. Clin. 13, 63-70. doi: 10.1016/j.jsmc.2017.09.006

McKeith, I. G., Boeve, B. F., Dickson, D. W., Halliday, G., Taylor, J. P., Weintraub, D., et al. (2017). Diagnosis and management of dementia with Lewy bodies: fourth consensus report of the DLB consortium. Neurology 89, 88-100. doi: 10.1212/WNL.0000000000004058

Menon, S. S., and Krishnamurthy, K. (2019). A comparison of static and dynamic functional connectivities for identifying subjects and biological sex using intrinsic individual brain connectivity. Sci. Rep. 9:5729. doi: 10.1038/s41598019-42090-42094

Miller, R. L., Yaesoubi, M., Turner, J. A., Mathalon, D., Preda, A., Pearlson, G., et al. (2016). Higher dimensional meta-state analysis reveals reduced resting fMRI connectivity dynamism in schizophrenia patients. PLoS One 11:e0149849. doi: 10.1371/journal.pone.0149849

Nalci, A., Rao, B. D., and Liu, T. T. (2019). Nuisance effects and the limitations of nuisance regression in dynamic functional connectivity fMRI. Neuroimage 184, 1005-1031. doi: 10.1016/j.neuroimage.2018.09.024

Olney, N. T., Spina, S., and Miller, B. L. (2017). Frontotemporal dementia. Neurol. Clin. 35, 339-374. doi: 10.1016/j.ncl.2017.01.008

Park, H. J., Friston, K. J., Pae, C., Park, B., and Razi, A. (2018). Dynamic effective connectivity in resting state fMRI. Neuroimage 180(Pt B), 594-608. doi: 10. 1016/j.neuroimage.2017.11.033

Peraza, L. R., Kaiser, M., Firbank, M., Graziadio, S., Bonanni, L., Onofrj, M., et al. (2014). fMRI resting state networks and their association with cognitive fluctuations in dementia with Lewy bodies. Neuroimage Clin. 4, 558-565. doi: 10.1016/j.nicl.2014.03.013

Petrella, J. R., Sheldon, F. C., Prince, S. E., Calhoun, V. D., and Doraiswamy, P. M. (2011). Default mode network connectivity in stable vs progressive mild cognitive impairment. Neurology 76, 511-517. doi: 10.1212/WNL. 0b013e31820af94e

Poewe, W., Seppi, K., Tanner, C. M., Halliday, G. M., Brundin, P., Volkmann, J., et al. (2017). Parkinson disease. Nat. Rev. Dis. Primers 3:17013. doi: 10.1038/ nrdp. 2017.13

Power, J. D., Mitra, A., Laumann, T. O., Snyder, A. Z., Schlaggar, B. L., and Petersen, S. E. (2014). Methods to detect, characterize, and remove motion artifact in resting state fMRI. Neuroimage 84, 320-341. doi: 10.1016/j.neuroimage.2013. 08.048

Power, J. D., Plitt, M., Gotts, S. J., Kundu, P., Voon, V., Bandettini, P. A., et al. (2018). Ridding fMRI data of motion-related influences: removal of signals with distinct spatial and physical bases in multiecho data. Proc. Natl. Acad Sci. U.S.A. 115, E2105-E2114. doi: 10.1073/pnas.1720985115

Premi, E., Calhoun, V. D., Diano, M., Gazzina, S., Cosseddu, M., Alberici, A., et al. (2019). The inner fluctuations of the brain in presymptomatic Frontotemporal Dementia: the chronnectome fingerprint. Neuroimage 189, 645-654. doi: 10.1016/j.neuroimage.2019.01.080

Preti, M. G., Bolton, T. A., and Van De Ville, D. (2017). The dynamic functional connectome: state-of-the-art and perspectives. Neuroimage 160, 41-54. doi: 10.1016/j.neuroimage.2016.12.061

Putcha, D., Ross, R. S., Cronin-Golomb, A., Janes, A. C., and Stern, C. E. (2015). Altered intrinsic functional coupling between core neurocognitive networks in Parkinson's disease. Neuroimage Clin. 7, 449-455. doi: 10.1016/j.nicl.2015. 01.012

Quevenco, F. C., Preti, M. G., van Bergen, J. M., Hua, J., Wyss, M., Li, X., et al. (2017). Memory performance-related dynamic brain connectivity indicates pathological burden and genetic risk for Alzheimer's disease. Alzheimers Res. Ther. 9:24. doi: 10.1186/s13195-017-0249-247

Rohrer, J. D., and Warren, J. D. (2011). Phenotypic signatures of genetic frontotemporal dementia. Curr. Opin. Neurol. 24, 542-549. doi: 10.1097/WCO. 0b013e32834cd442

Smith, S. M., Miller, K. L., Salimi-Khorshidi, G., Webster, M., Beckmann, C. F., Nichols, T. E., et al. (2011). Network modelling methods for FMRI. Neuroimage 54, 875-891. doi: 10.1016/j.neuroimage.2010.08.063 
Smitha, K. A., Akhil Raja, K., Arun, K. M., Rajesh, P. G., Thomas, B., Kapilamoorthy, T. R., et al. (2017). Resting state fMRI: a review on methods in resting state connectivity analysis and resting state networks. Neuroradiol. J. 30, 305-317. doi: 10.1177/1971400917697342

Sourty, M., Thoraval, L., Roquet, D., Armspach, J. P., Foucher, J., and Blanc, F. (2016). Identifying dynamic functional connectivity changes in dementia with lewy bodies based on product hidden markov models. Front. Comput. Neurosci. 10:60. doi: 10.3389/fncom.2016.00060

Tagliazucchi, E., and Laufs, H. (2014). Decoding wakefulness levels from typical fMRI resting-state data reveals reliable drifts between wakefulness and sleep. Neuron 82, 695-708. doi: 10.1016/j.neuron.2014.03.020

Tagliazucchi, E., von Wegner, F., Morzelewski, A., Brodbeck, V., and Laufs, H. (2012). Dynamic BOLD functional connectivity in humans and its electrophysiological correlates. Front. Hum. Neurosci. 6:339. doi: 10.3389/ fnhum.2012.00339

Tessitore, A., Esposito, F., Vitale, C., Santangelo, G., Amboni, M., Russo, A., et al. (2012). Default-mode network connectivity in cognitively unimpaired patients with Parkinson disease. Neurology 79, 2226-2232. doi: 10.1212/WNL. 0b013e31827689d6

van Eimeren, T., Monchi, O., Ballanger, B., and Strafella, A. P. (2009). Dysfunction of the default mode network in Parkinson disease: a functional magnetic resonance imaging study. Arch. Neurol. 66, 877-883. doi: 10.1001/archneurol. 2009.97

Wee, C. Y., Yang, S., Yap, P. T., Shen, D., and Alzheimer's Disease Neuroimaging Initiative (2016). Sparse temporally dynamic resting-state functional connectivity networks for early MCI identification. Brain Imaging Behav. 10, 342-356. doi: 10.1007/s11682-015-9408-9402

Xu, Y., and Lindquist, M. A. (2015). Dynamic connectivity detection: an algorithm for determining functional connectivity change points in fMRI data. Front. Neurosci. 9:285. doi: 10.3389/fnins.2015.00285
Zhan, Z. W., Lin, L. Z., Yu, E. H., Xin, J. W., Lin, L., Lin, H. L., et al. (2018). Abnormal resting-state functional connectivity in posterior cingulate cortex of Parkinson's disease with mild cognitive impairment and dementia. CNS Neurosci. Ther. 24, 897-905. doi: 10.1111/cns.12838

Zhuang, X., Walsh, R. R., Sreenivasan, K., Yang, Z., Mishra, V., and Cordes, D. (2018). Incorporating spatial constraint in co-activation pattern analysis to explore the dynamics of resting-state networks: an application to Parkinson's disease. Neuroimage 172, 64-84. doi: 10.1016/j.neuroimage.2018.01.019

Conflict of Interest Statement: MF is Editor-in-Chief of the Journal of Neurology; has received compensation for consulting services and/or speaking activities from Biogen Idec, Merck-Serono, Novartis, Teva Pharmaceutical Industries; and has received research support from Biogen Idec, Merck-Serono, Novartis, Teva Pharmaceutical Industries, Roche, Italian Ministry of Health, Fondazione Italiana Sclerosi Multipla, and ARiSLA (Fondazione Italiana di Ricerca per la SLA). FA is Section Editor of NeuroImage: Clinical; has received speaker honoraria from Biogen Idec and Novartis; and receives or has received research supports from the Italian Ministry of Health, AriSLA (Fondazione Italiana di Ricerca per la SLA), and the European Research Council.

The remaining authors declare that the research was conducted in the absence of any commercial or financial relationships that could be construed as a potential conflict of interest.

Copyright () 2019 Filippi, Spinelli, Cividini and Agosta. This is an open-access article distributed under the terms of the Creative Commons Attribution License (CC BY). The use, distribution or reproduction in other forums is permitted, provided the original author(s) and the copyright owner(s) are credited and that the original publication in this journal is cited, in accordance with accepted academic practice. No use, distribution or reproduction is permitted which does not comply with these terms. 